\title{
Red Giant Clump in the Tycho-2 Catalogue
}

\author{
G. A. Gontcharov* \\ July 11, 2018
}

Pulkovo Astronomical Observatory, Russian Academy of Sciences, Pulkovskoe sh. 65, St. Petersburg, 196140 Russia

Key words: Galaxy (Milky Way), spiral arms, stellar classification, types of stars, color-magnitude diagram.

The Tycho-2 proper motions and Tycho-2 and 2MASS photometry are used to select 97348 red giant clump (RGC) stars. The interstellar extinction and photometric distance are calculated for each of the stars. The selected stars are shown to form a selection-unbiased sample of RGC stars within about $350 \mathrm{pc}$ of the Sun with the addition of more distant stars. The distribution of the selected stars in space and their motion are consistent with the assumption that the RGC contains Galactic disk stars with various ages and metallicities, including a significant fraction of stars younger than $1 \mathrm{Gyr}$ with masses of more than $2 M_{\odot}$. These young stars show differences of their statistical characteristics from those of older RGC stars, including differences in the variations of their distribution density with distance from the Galactic plane and in the dispersion of their velocities found using radial velocities and proper motions. The Sun has been found to rise above the Galactic plane by $13 \pm 1 \mathrm{pc}$. The distribution density of the stars under consideration in space is probably determined by the Local Spiral Arm and the distribution of absorbing matter in the plane of the Gould Belt.

\footnotetext{
*E-mail: georgegontcharov@yahoo.com
} 


\section{INTRODUCTION}

A region of enhanced distribution density of red giants called the red giant clump (RGC) is clearly seen on the color-absolute magnitude diagrams for open clusters and various samples of stars. Since these stars have a specific absolute magnitude, their photometric distances can be determined rather accurately.

In globular clusters, the horizontal branch (HB) occupies approximately the same region of the color-absolute magnitude diagram. However, in contrast to the $\mathrm{HB}$, the RGC is not an evolutionally homogeneous group of stars and consists of three groups considered below that are located in this region of the diagram for various reasons.

(1) It is traditionally believed that low-mass core helium burning stars after the red giant branch (RGB) phase and a helium flash constitute an overwhelming majority of the RGC (and HB) stars (Girardi et al. 1998). Before the flash, the mass of such a star is lower than some limiting value, $\mathrm{M}_{H e F} \approx 2$ $\mathrm{M} \odot$, and the helium core is degenerate. After the flash, the degeneracy is removed and the luminosity of these helium burning stars is almost independent of the color index and age, since the helium core mass at this time is almost independent of the stellar mass. However, the color index depends on the mass, age, and metallicity: it decreases with decreasing metallicity and decreasing age (increasing mass) (Girardi 1999). Nevertheless, if the metallicity has increased appreciably over the lifetime of the Galactic disk, these dependences almost cancel each other out and the stars under consideration should have a moderately large spread in color (Girardi et al. 1998). The main-sequence (MS) progenitors of these stars are F and early-G stars, because later-type stars could not fall into the RGC over the lifetime of the Galactic disk $\left(<10^{10} \mathrm{yr}\right)$. Since these stars are fairly old by the helium flash, below we call them RGC old stars (RGC-O). A typical Galactic disk star with a mass of $1.2 M_{\odot}$ and a solar metallicity $(\mathbf{Z}=0.02, \mathbf{Y}=0.27)$ spends $\approx 6 \times 10^{9} \mathrm{yr}$ on the MS, $0.4 \times 10^{9} \mathrm{yr}$ as a subgiant, $0.6 \times 10^{9} \mathrm{yr}$ on the RGB, and only $0.1 \times 10^{9} \mathrm{yr}$ in the RGC (Castelliani et al. 1992; Girardi et al. 1998). Thus, the clump of these stars in the RGC is formed only because the RGB stars with a large spread in absolute magnitude and color $\left(-2<M_{V}<4\right.$, $\left.0.7^{m}<(B-V)<1.6^{m}\right)$ concentrate for a certain time in the RGC with a small spread $\left(0.5<M_{V}<1,0.8^{m}<(B-V)<1.2^{m}\right)$. By comparing

these ranges and the residence times on the RGB and in the RGC, it may be concluded that the distribution density of the RGC stars should be higher 
than that of the RGB ones only a factor of $2-3$. Such are the diagrams for globular clusters. In contrast, in the solar neighborhood, i.e., in the Galactic disk, the RGC is much more densely populated than the RGB. Once the Hipparcos Catalogue (ESA 1997) has been published, it has become clear that the distribution density of the RGC stars with respect to the RGB is appreciably higher than that predicted by the theory (Girardi et al. 1998). This can be partly explained theoretically by the fact that low-mass metalpoor stars fall into the RGC relatively quickly: a star with $\mathrm{M}=1.5 M_{\odot}$ and $\mathbf{Z}=0.001$ falls into the $\mathrm{RGC} \approx 2$ Gyr after its birth (Girardi et al. 2000). In addition, Girardi et al. (2005) showed that young, relatively massive stars theoretically could constitute an appreciable fraction of the RGC in spiral arms and other regions where the stars have been born for the last 1 Gyr.

(2) Thus, high-mass $\left(M>M_{H e F}\right)$ core helium burning stars after a fairly short hydrogen shell burning phase of a nondegenerate helium core also fall into the RGC. Since these stars are rather young by helium ignition, below we call them RGC young stars (RGC-Y). Their luminosity depends on the helium core mass, while the latter, in turn, depends on the stellar mass. However, the fraction of stars with masses higher than $3 M_{\odot}$, their total lifetime, and the residence time in the RGC are so small that, in fact, they are lost in samples with less massive stars and are absent in the RGC (Girardi 1999). As a result, the spread in luminosity and absolute magnitude for RGCY dominated by stars with $M \approx 2 M_{\odot}$ is only slightly larger than that for RGC-O. In addition, being young, RGC-Y have a small spread in metallicity and, therefore, occupy a compact region of the color-absolute magnitude diagram at the blue edge of the RGC in all samples of Galactic disk stars (Girardi et al. 1998). It should be noted that RGC-Y concentrate in the $\mathrm{RGC}$ region for a different reason than $\mathrm{RGC-O}$ : the core helium burning time for RGC-Y is considerably longer (about $200 \mathrm{Myr}$ ) and is shorter than the residence time of such a star on the MS, where its type is B or A, only by a factor of several. The ratio of the helium burning time to the hydrogen burning time and, accordingly, the ratio of the distribution densities of the RGC and MS stars is at a maximum for stars with a mass of about $2 M_{\odot}$ (Bressan et al. 1993).

(3) Some of the RGB stars can have the same color and absolute magnitude as RGC-O and RGCY, although they are at a different evolutionary phase and should be considered as a foreign admixture in the samples of RGC stars.

The fraction of these three groups in the RGC affects the mean abso- 
lute magnitude of the RGC for various samples of stars and, accordingly, the photometric distances (Girardi et al. 1998). In addition, many of the observed differences between globular and open clusters are determined by the large fraction of RGC-Y among the giants of open clusters (Girardi and Bertelli 1998). In clusters younger than 1 Gyr, the RGC should include only RGC-Y. Analysis of the RGC composition and properties of these stars is also important in testing the theory of stellar evolution and, in particular, in analyzing the metallicity dependence of the critical mass $M_{\mathrm{HeF}}$ and modeling the helium flash and its consequences. This paper is one of the stages in investigating the RGC.

The fraction of RGC-Y and RGC-O for the nearest part of the Galactic disk can be estimated by selecting RGC stars from the Hipparcos and Tycho2 Catalogues (Høg et al. 2000) using $J, H$, and $K s$ infrared photometry from the 2MASS Catalogue (Skrutskie et al. 2006) and by analyzing their distribution in space and velocity dispersion. A similar study of O-B stars using proper motions and multiband photometry was performed previously (Gontcharov 2008). However, the much smaller spread in absolute magnitude for the RGC stars allows us to use their proper motions only to select stars by calculating their distances only from photometric data.

It should be noted that, being magnitude-limited, the Hipparcos and Tycho-2 Catalogues cannot contain any RGC stars outside the Galactic disk (in the bulge, halo, etc.): the RGC stars brighter than $V=11^{m}$ at an extinction as high as $1^{m}$ are closer than $1.5 \mathrm{kpc}$.

\section{RGC STARS IN THE HIPPARCOS CATA- LOGUE}

Following Girardi et al. (2005), let us compare the observed and theoretical color-absolute magnitude diagrams for 16726 stars from the Hipparcos Catalogue with the most accurate data. The proper motion components for these stars are known with an accuracy better than $0.002 \operatorname{arcsec} \mathrm{yr}^{-1}$, the relative error in the parallax is less than $10 \%$, the Hipparcos photometric magnitudes $B_{T}$ and $V_{T}$ are known with an accuracy better than $0.1^{m}$, the stars are neither double nor multiple, and their spectral classification is known. Since they are no more than $250 \mathrm{pc}$ away, their reddening is small and the absolute magnitudes calculated from their parallaxes will have no systematic errors. 
Figure 1a shows the positions of giants and MS stars of late spectral types among these stars on the $(B-V)-M_{V}$ diagram based on Hipparcos data. Theoretically, according to Girardi et al. (2005), RGC-O with an age from $2 \times 10^{9}$ to $10 \times 10^{9}$ yr and metallicity $0.004<\mathbf{Z}<0.020$ should occupy the region marked by the oval (the more metal-poor and, accordingly, older stars are located leftward); the RGB with the same age and metallicity should occupy the region between the two dotted lines (again, the more metal-poor and, accordingly older stars are located leftward); and RGC-Y with an age up to $2 \times 10^{9}$ yr and metallicity $0.006<\mathbf{Z}<0.020$ should occupy the region marked by the nearly rectangular figure (the more metal-poor stars are located leftward; no age gradient is seen). The distribution of stars within the shown regions and the sharp decrease in distribution density outside them confirm the conclusion by Girardi et al. (2005) that RGC-Y and RGB stars constitute a significant fraction of the RGC stars in the nearest part of the Galactic disk and are located on the diagram precisely as predicted by the theory. Since the RGC in the Tycho-2 Catalogue also contains mostly disk stars within the nearest kiloparsec, it can be assumed that the color - absolute magnitude diagram for the Tycho-2 data and the conclusions drawn from it are similar to the results obtained from Hipparcos data.

Having assumed a uniform distribution of the RGC stars in age from $1 \pm 0.1$ to $10 \pm 2$ Gyr and in metallicity $\mathbf{Z}$ from $0.004 \pm 0.001$ to $0.020 \pm$ 0.001 (Bertelli et al. 2008; Girardi and Salaris 2001; Salaris and Girardi 2002), we calculate the theoretical mean absolute magnitudes of the RGC stars and their uncertainties due to these variations in initial parameters: $\overline{M_{B_{T}}}=2.06^{m} \pm 0.05^{m}, \overline{M_{V_{T}}}=0.86^{m} \pm 0.05^{m}, \overline{M_{J}}=-0.87^{m} \pm 0.05^{m}, \overline{M_{H}}=$ $-1.41^{m} \pm 0.05^{m}, \overline{M_{K s}}=-1.52^{m} \pm 0.05^{m}$. The theoretical color dependences of the absolute magnitudes calculated in the same way differ from the empirical ones obtained for all of the Hipparcos RGC stars located in the regions of the $(B-V)-M_{V}$ diagram marked in Fig. 1a. This difference results from the Hipparcos selection in favor of more luminous stars, among which relatively blue RGC-Y dominate in our case. This selection also has an effect on the mean absolute magnitudes but within the above uncertainty of $0.05^{\mathrm{m}}$. At the same time, the theoretical and empirical coefficients of the color dependences differ by up to 30\%, because brighter RGC-Y are simultaneously bluer. Since this selection should also manifest itself in the Tycho-2 data, as in any magnitude-limited sample of disk stars, below we 
use the empirical dependences

$$
M_{B_{T}}=2.89\left(B_{T}-V_{T}\right)-1.42, M_{J}=1.25(J-K s)-1.68 .
$$

The standard deviation of the absolute magnitudes for the RGC stars from the Hipparcos data after applying a correction for the color dependence is $0.4^{m}$. This is an estimate of the extent to which we err by replacing the individual absolute magnitudes with the mean color dependences.

\section{EXTINCTION AND DISTANCE CALCULA- TIONS}

Using several photometric magnitudes of a star in the RGC, we can determine the extinction $A_{V}$, reddening $E_{(B-V)}$, and unreddened $(B-V)_{0}$ color index for the latter. It is well known that

$$
5 \log (r)-5=B_{T}-M_{B_{T}}-A_{B_{T}}=J-M_{J}-A_{J},
$$

where $\mathrm{r}$ is the distance, $A_{B_{T}}, A_{J}$ are the interstellar extinctions for the corresponding magnitudes (other magnitudes can also be used). We use the dependence of extinction $A_{\lambda}$ on the effective wavelength $\lambda$ to reduce all extinctions to the same $A_{V}$. This dependence is analyzed in detail in many studies, whose results give the difference between the visible and infrared extinctions with a relative accuracy of better than $5 \%$ used below (see, e.g., Nishiyama et al. 2006; Marshall et al. 2006; and references therein). Having analyzed these data by taking into account the effective wavelengths $\lambda_{V}=0.553, \lambda_{B_{T}}=0.435, \lambda_{J}=1.24 \lambda_{K s}=2.16$ microns, we adopted

$$
A_{B_{T}}=1.32 A_{V}, A_{J}=0.28 A_{V}, A_{K s}=0.11 A_{V} .
$$

Substituting (1) and (3) into (2) yields an estimate of the interstellar extinction for each star:

$$
A_{V}=\left(B_{T}-2.89\left(B_{T}-V_{T}\right)+1.42-J+1.25(J-K s)-1.68\right) / 1.04
$$

For the Hipparcos RGC stars, we found

$$
(B-V)=0.78\left(B_{T}-V_{T}\right)
$$


Taking $E_{(B-V)}=A_{V} / 3$, we calculate the unreddened color index

$$
(B-V)_{0}=0.78\left(B_{T}-V_{T}\right)-A_{V} / 3 .
$$

Negative extinctions were also used. Replacing them by zeroes changes the composition of the sample by less than $1 \%$.

The photometric distances were calculated from the formula

$$
\log (r)=\left(5+K s+1.52-0.11 A_{V}\right) / 5,
$$

where $r$ is the sought-for distance. We also used negative extinctions, which does not affect the result, there are no stars with an extinction $A_{V}<-0.35^{m}$ in the final sample. For the selected stars (see below), we calculated the Galactic rectangular coordinates $X Y Z$, where $X$ increases toward the Galactic center, $Y$ increases in the direction of Galactic rotation, and $Z$ increases toward the Galactic North Pole.

It should be noted that, in general, the derived extinction and photometric distance are valid only for the RGC stars due to the replacement of the individual absolute magnitude by its mean color dependence (1). Therefore, the proper selection of RGC stars discussed below is important.

\section{THE SELECTION OF STARS}

When the RGC stars are selected from the Tycho-2 Catalogue, it is first necessary to eliminate the variable and binary stars as well as the stars with inaccurate initial data: we rejected the variables (designated as $\mathrm{G}, \mathrm{N}$ in field T47 or U, V, W in Tycho field T48) and binaries (designated as D, R, S, Y, Z in Tycho field T49) marked in the Tycho Catalogue (ESA 1997, vol. 1, pp. 156-158), including the photocenters of stellar pairs and the stars with components closer than 25 arcsec (Tycho-2 fields pflag and poflg are not blank, field prox $<250)$ (Høg et al. 2000), the stars whose $\left(B_{T}-V_{T}\right)$ had an accuracy lower than $0.15^{m}$, while $(J-K s)$ and $(J-H)$ had an accuracy lower than $0.05^{m}$, and the stars for which at least one of the proper-motion components had an accuracy lower than $0.004 \operatorname{arcsec}_{\mathrm{yr}^{-1}}$. In addition, imposing reasonable, but fairly wide constraints on the color indices allowed the binary stars with incorrectly identified components to be rejected.

The RGC stars are usually selected from the 2MASS Catalogue by their positions on the color - apparent magnitude diagram (Cabrera-Lavers et 
al. 2007; and references therein). However, the analysis by Marshall et al. (2006) showed that distant RGC stars are effectively separated from close MS dwarfs of the same color only in samples of rather faint stars close to the Galactic plane owing to their distinctly different distances and reddenings: at $V=14^{m}$, an RGC star with $M_{V} \approx 1$ is located at a heliocentric distance of several kpc and should redden strongly, as distinct from a MS dwarf with $M_{V} \approx 6$ located at a heliocentric distance of $\approx 400$ pc. For the Tycho- 2 stars located mostly within the nearest kiloparsec, using the apparent magnitude does not allow RGC stars to be separated from dwarfs (Marshall et al. 2006).

They can be separated using the reduced proper motion, which is a substitute for the absolute magnitude in the absence of systematic stellar motions and observational selection:

$$
M_{V}^{\prime}=V+5+5 \log (\mu)
$$

where $\mu=\left(\mu_{\alpha}^{2} \cos \delta+\mu_{\delta}^{2}\right)^{1 / 2}$ is the proper motion in arcsecs (Parenago 1954).

The proper motions used to calculate $M_{V}^{\prime}$ were corrected for the Galactic rotation and solar motion to the apex using the photometric distances calculated from (7). For the initial selection of RGC stars, we adopted typical values for the constants of Galactic rotation $(A=+15, B=-10, C=0$, $\left.K=0 \mathrm{~km} \mathrm{~s}^{-1} \mathrm{kpc}^{-1}\right)$ and solar motion to the apex $\left(V=19.5 \mathrm{~km} \mathrm{~s}^{-1}\right.$, $L=57^{\circ}, B=22^{\circ}$ ) (Parenago 1954). Subsequently, for the selected stars with radial velocities from the Pulkovo Compilation of Radial Velocities (PCRV) (Gontcharov 2006), we calculated the velocity components $U, V, W$ in the Galactic coordinate system in $\mathrm{km} \mathrm{s}^{-1}$ and used them to recalculate the constants of Galactic rotation and solar motion to the apex; subsequently, we corrected $M_{V}^{\prime}$ and used them to reselect the stars. After the second iteration, the calculated parameters of Galactic rotation and solar motion to the apex and the list of selected stars did not change. The derived constants of Galactic rotation $(A=+15.5 \pm 2, B=-11.3 \pm 2, C=-4.7 \pm 2, K=-3.4 \pm 2$ $\mathrm{km} \mathrm{s}^{-1} \mathrm{kpc}^{-1}$ ) and solar motion to the apex relative to the centroid of the selected stars $\left(U_{\odot}=+8.4 \pm 0.5, V_{\odot}=+17.6 \pm 0.3, W_{\odot}=+2.7 \pm 0.4 \mathrm{~km}\right.$ $\mathrm{s}^{-1}$, respectively, $\left.\mathrm{V}=19.7 \mathrm{~km} \mathrm{~s}^{-1}, L=64.5^{\circ}, B=7.9^{\circ}\right)$ agree with those universally accepted for the disk stars.

Figure $1 \mathrm{~b}$ shows the positions of the same Hipparcos giants and MS stars as those in Fig. 1a but on the $(B-V)$ - reduced proper motion $M_{V}^{\prime}$ diagram. We see that the RGC is blurred but fairly isolated from the bright giants, subgiants, and dwarfs. Figures 1c and 1d show similar diagrams for Tycho-2 
stars in a typical sky region before and after dereddening, respectively. We see that there are almost no dwarfs of the same color as the RGC among the Tycho-2 stars. As in Hipparcos, the RGC in Tycho-2 is identified as a region of enhanced distribution density of stars. In Fig. 1d, the region within the distribution density isoline corresponding to the maximum density gradient is shaded. In the same figure, the two ellipses that describe best this isoline indicate the adopted region all stars of which were selected as presumed RGC stars and they are considered below. The left and right ellipses roughly reflect the distributions of RGC-Y and RGC-O, respectively. Comparison of the regions marked in Figs. 1a and 1d shows that the ellipses are located in the region of theoretically predicted color indices. The maximum of the distribution of RGC stars is $(B-V)_{0}=0.92^{m}, M_{V}^{\prime}=5$. The centers and radii of the ellipses for $\mathrm{RGC}-\mathrm{Y}$ and $\mathrm{RGC}-\mathrm{O}$ are

$$
\begin{aligned}
& (B-V)_{0}=0.90^{m} \pm 0.13^{m}, M_{V}^{\prime}=4.7 \pm 2.1, \\
& (B-V)_{0}=0.98^{m} \pm 0.20^{m}, M_{V}^{\prime}=5.1 \pm 1.3 .
\end{aligned}
$$

These quantities do not depend on the Galactic coordinates.

For bright stars $\left(J<5^{m}\right)$, the 2MASS infrared photometry is inaccurate and many of the bright (close) RGC stars selected by the described method have erroneous color indices and should be rejected. This is how a fictitious region of reduced distribution density of stars within about $150 \mathrm{pc}$ of the Sun emerges. To avoid this, we selected the bright stars directly from Hipparcos when the following conditions were satisfied: (1) $J<5^{m}, H<5^{m}, K s<5^{m}$; (2) the accuracy of the IR magnitudes used is lower than $0.05^{m}$; (3) $\pi>$ $0.005^{\prime}$; and (4) $-1.5^{m}<M_{V_{T}}<1.3^{m}$ for $0.75^{m}<(B-V)_{0}<1.02^{m}$ or $0.5^{m}<M_{V_{T}}<1.5^{m}$ for $1.02^{m}<(B-V)_{0}<1.20^{m}$. We used the extinction from the model by Arenou et al. (1992). There were 1496 such stars. Below, we use their trigonometric distances.

We can see how efficient the use of the reduced proper motions for the selection of RGC stars is from the following Monte Carlo simulations. We specify the distributions of subgiant, RGB, RGC, and dwarf stars with color indices $0.77^{m}<(B-V)_{0}<1.2^{m}$ in space velocity $V$ and apparent and absolute magnitudes $V$ and $M_{V}$. Using the equations

$$
\begin{gathered}
5 \log (r)-5=V-M_{V}, \\
\mu=V / 4.74 r
\end{gathered}
$$


Eq. (8), and the assumption about an initially uniform selection-biased distribution of stars in space, we then obtain the distributions of subgiant, RGB, RGC, and dwarf stars from the reduced proper motion $M_{V}^{\prime}$. A detailed discussion of the simulations and the use of the reduced proper motions in various studies deserves a separate paper. The main simulation results are the following.

G-M dwarfs are almost absent in Tycho-2 and among the selected stars. The main reason is that this catalogue is magnitude-limited and, accordingly, the volume of space where the dwarfs are selected is small, no father than 150 pc. Dwarfs probably constitute a majority among the selected stars with a true distance up to $150 \mathrm{pc}$, but, according to our simulations, fewer than 500 stars $(<0.5 \%$ of the entire sample; for comparison, 1233 RGC stars within 150 pc were selected directly) should be selected in this region of space based on photometry and $M_{V}^{\prime}$. Since the absolute magnitudes erroneously adopted for the dwarfs (just as for the RGC stars) are several magnitudes brighter than the true ones, their photometric distances are larger than the true ones by a factor of several. As a result, these dwarfs should be a small admixture $(<1 \%)$ that is rather uniformly distributed in photometric distance and that does not produce any biases in the results (not the dwarfs, but the RGC stars selected directly dominate even near the Sun).

RGB stars and subgiants can initially account for up to $20 \%$ of the selected stars. Since the adopted absolute magnitudes and extinctions do not correspond to the true ones, most of the subgiants and many of the RGB stars have negative extinctions and photometric distances of more than $500 \mathrm{pc}$, while some of the RGB stars have high extinctions at small distances. To reduce the fraction of these stars, we rejected the stars with $A_{V}>0.6^{m} /(|\sin (b)|+0.18)$, where $b$ is the Galactic latitude. This limit is a reasonable constraint, since it approximately twice the mean extinction for a given latitude determined from the model by Arenou et al. (1992) within $1 \mathrm{kpc}$ and is $3.3^{m}$ near the Galactic equator. In addition, because of the color difference between the RGC stars and subgiants, the latter are largely eliminated by the constraint $V_{T}-K s>0.75\left(B_{T}-J\right)+0.05$. The remaining subgiants should have photometric distances that are mostly larger than 500 pc. As a result, according to our simulations, the selected set of presumed RGC stars within 500 pc should contain less than $10 \%$ of the RGB stars and subgiants and less than $1 \%$ of the dwarfs.

These conclusions are confirmed by the statistics of the selected stars. For 20820 selected stars, there is a spectral classification in the Tycho Spectral 
Types (TST) Catalogue (Wright et al. 2003), including the luminosity class. Among them, there are 18505 (89\%) G5III-M2III giants (the RGC and part of the RGB, given the classification errors), 281 (1.3\%) GV-MV dwarfs, 1298 (6\%) GIV-KIV subgiants, 633 (3\%) bright giants and supergiants, and 103 $(0.7 \%)$ other stars.

We selected a total of 97348 presumed RGC stars from about 2.5 million Tycho-2 stars. 14291 selected stars are contained in Hipparcos and 45813 are contained in the TST Catalogue; the spectral classification of 51412 selected stars $(53 \%)$ has not been known to the present day.

The selected stars have fairly accurate proper motions and photometry: for $85 \%$ of the stars, $\mu_{\alpha} \cos \delta$ and $\mu_{\delta}$ are known to within $0.003 \operatorname{arcsec} \mathrm{yr}^{-1}$, $B_{T}$ and $V_{T}$ are known to within $0.15^{m}$ and $0.1^{m}$, respectively, and the infrared magnitudes are known to within $0.05^{\mathrm{m}}$.

The selection ellipses specified by conditions (9) and (10) contain approximately equal numbers of stars. However, the fraction of RGC-Y and RGC-O is difficult to estimate accurately, since the zone of intersection between the ellipses is large and, in addition, the admixture of foreign stars is distributed nonuniformly. Only the fact that RGC-Y account for a significant fraction (possibly up to half) of the RGC stars is beyond question. Below, we consider various characteristics of the selected stars by dividing the sample into two equal parts, depending on whether a star is closer to the center of one or the other ellipse on the $(B-V)_{0}-M_{V}^{\prime}$ diagram. Below, these groups of stars are arbitrarily called RGC-Y and RGC-O.

\section{THE DISTRIBUTION OF RGC STARS IN SPACE}

Figure 2 shows the distribution of the selected stars on the celestial sphere in Galactic coordinates. The distributions of RGC-Y and RGC-O are identical. Regions of reduced density, "voids", are clearly seen not far from the Galactic equator or, more precisely, along the great circle inclined to the equator by about $15^{\circ}$ and coincident with the equatorial plane of the Gould Belt. Obviously, these voids result from relatively strong absorption by the matter located within several hundred parsecs of the Sun in layers parallel to the equatorial plane of the Gould Belt.

Figure 3a compares the photometric distances with the distances calcu- 
lated from the Hipparcos parallaxes for 13079 stars with $\pi>0.001$ arcsec (RGC-Y and RGC-O are shown together). We see agreement between the distances within $350 \mathrm{pc}$ (apart from the stars selected directly), where the Hipparcos parallaxes are fairly accurate. The ratio of the standard deviation of the difference between the photometric and astrometric distances to the astrometric distance, $\sigma\left(r_{p h}-r_{H I P}\right) / r_{H I P}$, for the Hipparcos stars with 350 pc is 0.35 .

As we see from Fig. 3a, the photometric distances for some of the stars are appreciably smaller than the distances determined from the Hipparcos parallaxes. For these stars, the extinction calculated here is considerably lower than that corresponding to the Hipparcos parallax. This can be seen from Fig. 3b, which compares the derived extinction $A_{V}$ with that calculated by Arenou et al. (1992) using the Hipparcos parallaxes (RGC-Y and RGC-O are shown together). Good agreement for most of the stars suggests that the proposed method of correction for the extinction is applicable.

Since the reduced proper motions were used here only for the selection of stars, but not for the calculation of distances, in contrast to our previous study (Gontcharov 2008), the derived distances will not have the corresponding systematic errors. In addition, the systematic errors that arise when the trigonometric parallaxes are used to calculate the distances do not arise here either. The accuracy of the derived distances is determined by the accuracy of the initial photometric quantities, the spread in absolute magnitude for the RGC stars, the fraction of stars with a different absolute magnitude, the error in the adopted absorption coefficient, and other factors. The estimates of these uncertainties made previously allow us to estimate the relative accuracy of the derived photometric distances, about $25 \%$ (corresponding to a photometric error of $0.5^{\mathrm{m}}$ ). Thus, for distances larger than $350 \mathrm{pc}$, the photometric distances should be more accurate than the distances determined from the Hipparcos parallaxes.

In Fig. 4, the extinction $A_{V}$ is plotted against the distance for (a) RGC-Y and (b) RGC-O. We see the mean extinction to increase up to a distance of about 550 and 450 pc, respectively (the right white dotted line). Further out, the mean extinction decreases. This results from selection, because the catalog is magnitude-limited (Gontcharov 2008). Far from the Galactic plane, the RGC-Y and RGC-O subsamples are selection-unbiased up to these distances. Near the Galactic plane, the corresponding distance is determined from the maximum extinctions, which occur at a distance of about $350 \mathrm{pc}$ marked by the left white dotted line for both subsamples. As expected, 
RGC-Y are seen at larger heliocentric distances because of their higher mean luminosities. There are more stars with negative extinctions among RGC-O, which is due probably to the larger admixture of RGB stars and subgiants.

Jointly using the 2MASS and Tycho-2 Catalogues allows a sample of RGC stars to be considered in a larger region of space than that using Hipparcos. This can be seen from Fig. 5, which shows the spatial distribution of stars in projection onto the $X Y, X Z$, and $Y Z$ planes: (a) calculated from the Hipparcos parallaxes for 13889 selected presumed RGC stars with positive Hipparcos parallaxes; (b) calculated from the photometric distances all 97 348 selected RGC stars; (c) the same indicated by a contour map for half of the selected conditional RGC-Y; (d) the same for conditional RGC-O.

We see that the Hipparcos RGC stars are distributed uniformly in space within about $200 \mathrm{pc}$. It is virtually impossible to analyze the distribution of RGC stars outside this volume on the basis of Hipparcos data. In contrast to Hipparcos, our sample of presumed RGC stars reveals a decrease in the distribution density of stars with distance from the Galactic plane (the plots in the $X Z$ and $Y Z$ planes), with this decrease being slightly different for RGC-Y and RGC-O. On the same plots, we see the role of extinction in "withdrawing" the stars near the Galactic plane; in the first and second Galactic quadrants, this role is appreciably greater than that in the third and fourth ones, which manifests itself in the mean $Y$ of the stars under consideration: $\bar{Y}=-25 \pm 1 \mathrm{pc}$. The positions of the "holes" agree with the orientation of the Gould Belt.

Our sample of stars is appreciably more extended along the $Y$ axis than along the $X$ axis, which is completely invisible in the Hipparcos sample. The Local Spiral Arm of the Galaxy stretching along the $Y$ axis probably manifests itself in this way. In the Galactic center-anticenter direction, the Sun is located almost at the center of the spatial distribution of the sample of stars under consideration: $\bar{X}=13 \pm 1$ pc. Given a noticeable decrease in density in these directions at a distance of more than $500 \mathrm{pc}$, which is probably caused not by selection, but by the location outside the Local Arm, it may be concluded that the Sun lies approximately at the center of the Local Arm section.

The distribution density of 39927 selected presumed RGC stars in the cylindrical region of space $\left(X^{2}+Y^{2}\right)^{1 / 2}<400$ pc does not depend on $X$ and $Y$, but depends only on $Z$. Thus, we are probably dealing with a selectionunbiased sample of RGC stars in the vertical cylindrical section of the Galactic disk in the solar neighborhood. The mean value of $\mathrm{Z}$ for the stars in the 
cylinder of space under consideration suggests that the Sun rises above the Galactic plane by $13 \pm 1$ pc. This also manifests itself in the asymmetric distribution of stars in the cylinder under consideration along the $Z$ axis, which is shown in Fig. 6 by the columns for each 100 pc for (a) conditional RGC-Y and (b) RGC-O: at all of the distances, except $|Z| \approx 700$ and $|Z|>900 \mathrm{pc}$, the number of stars in each column with positive $Z$ is smaller than that in the column with $-Z$.

Theoretically, the distribution density of the stars can decrease with distance $\mathrm{Z}$ as the square of the hyperbolic secant $\operatorname{sech}^{2}\left(2 Z / Z_{0}\right)$ (Girardi et al. $2005)$ or according to the barometric law $D_{0} \cdot e^{-|Z| / Z_{0}}$, where $D_{0}$ is the density in the equatorial plane, which does not necessarily coincide with the Galactic plane, and $Z_{0}$ is the distance from this plane at which the density decreases by a factor of $e$ or the half-thickness of a homogeneous layer of stars (Parenago 1954, p. 264). The solid curves in Figs. 6a and 6b indicate the functions $4000 e^{-\left|Z+13^{\circ}\right| / 220}$ and $4900 e^{-\left|Z+13^{\circ}\right| / 230}$ and the dotted curves indicate $13800 /\left(e^{\left(Z+13^{\circ}\right) / 280}+e^{-\left(Z+13^{\circ}\right) / 280}\right)$ and $16800 /\left(e^{\left(Z+13^{\circ}\right) / 280}+e^{-\left(Z+13^{\circ}\right) / 280}\right)$, whose coefficients were found by the least-squares method. The distributions of RGC-Y and RGC-O are better described by the barometric law and the square of the hyperbolic secant, respectively. The values of $Z_{0}$ found are typical of the Galactic thin-disk stars.

\section{THE KINEMATICS OF RGC STARS}

For 4163 selected stars with radial velocities from the PCRV and RAVE catalogs (Steinmetz et al. 2006), we calculated the velocity components $U$, $V, W$ in the Galactic coordinate system in $\mathrm{km} \mathrm{s}^{-1}$. These stars include the 1990 stars considered by Famaey et al. (2005), but, in contrast to them, they are distributed fairly uniformly over the sky. These data allow some general conclusions about the kinematics of the selected presumed RGC stars to be reached (a detailed analysis will be presented in a separate publication).

Above, we mentioned the parameters of Galactic rotation and solar motion to the apex calculated from these data and consistent with those universally accepted for disk stars.

Figure 7 shows the distribution of the 4163 stars under consideration in velocity components $U$ and $V$ : (a) the 900 stars closest to the center of the RGC-Y ellipse and farthest from the center of the RGC-O ellipse on the $(B-V)_{0}-M_{V}^{\prime}$ diagram, i.e., presumably the youngest and most massive 
stars of the sample and (b) the remaining stars. Comparison with the results of Famaey et al. (2005) suggests that subsample (a) actually contains stars younger than 1 Gyr, while subsample (b) contains typical disk stars of various ages. Thus, it is confirmed that the RGC contains a significant fraction (probably $>20 \%$ ) of relatively young massive stars.

For both RGC-Y and RGC-O, the dispersion of the velocity component $\mathrm{W}$ increases with distance from the Galactic plane, on average, from $18 \mathrm{~km}$ $\mathrm{s}^{-1}$ in the plane to $30 \mathrm{~km} \mathrm{~s}^{-1}$ at $|Z|=500 \mathrm{pc}$. The deviations from a monotonic change in dispersion observed at some $Z$ require a separate study.

We found contraction of the set of stars under considerations along the $Z$ axis, i.e., a velocity gradient $W / Z=-16 \pm 4 W / Z=-12 \pm 4 \mathrm{~km} \mathrm{~s}^{-1} \mathrm{kpc}^{-1}$ for RGC-Y and RGC-O, respectively.

\section{CONCLUSIONS}

This study has shown that the 2MASS and Tycho-2 broad band multicolor photometry and proper motions are sufficient to calculate the individual interstellar extinctions and photometric distances for RGC stars. Using the method under consideration, we selected the stars in a region of space that exceeded a similar region for the Hipparcos Catalogue by a factor of several. Within about 350 pc of the Sun, the sample will be selection-unbiased. This allowed us to detect a decrease in the distribution density of stars both with height above the Galactic plane and at the presumed edges of the Local Spiral Arm and to determine the rise of the Sun above the Galactic plane with a high accuracy. In the region of space under consideration, the RGC was shown to be a mixture of three groups of stars, including a significant fraction of stars younger than 1 Gyr with masses higher than $2 M_{\odot}$.

The method under consideration is important for investigating Galactic structures and for modern large astronomical projects, whose key objective is an automatic classification of millions of stars and extended objects based on their multicolor photometry.

\section{ACKNOWLEDGMENTS}

I wish to thank the referee for helpful remarks. In this study, I used the 2MASS (Two Micron All Sky Survey) Catalogue, which is a joint project 
of the Massachusetts University and the IR Data Reduction and Analysis Center of the California Institute of Technology financed by NASA and the National Science Foundation. I also used resources from the Strasbourg Data Center (France) (http://cdsweb.u-strasbg.fr/). This study was supported by the Russian Foundation for Basic Research (http://www.rfbr.ru) (project nos. 05-02-17047 and 08-02-00400).

\section{References}

1. F. Arenou, M. Grenon, A. Gomez, Astron. Astrophys. 258, 104 (1992).

2. G. Bertelli, L. Girardi, P. Marigo, et al., Astron. Astrophys. 484, 815 (2008).

3. A. Bressan, F. Fagotto, G. Bertelli, et al., Astron. Astrophys. Suppl. Ser. 100, 647 (1993).

4. A. Cabrera-Lavers, P.L. Hammersley, C. Gonzalez-Fernandez, et al., Astron. Astrophys. 465, 825 (2007).

5. V. Castelliani, A. Chieffi, O. Straniero, Astrophys. J. Suppl. Ser. 78, 517 (1992).

6. ESA, Hipparcos and Tycho catalogues (ESA, 1997).

7. B. Famaey, A. Jorissen, X. Luri, et al., Astron. Astrophys. 430, 165 (2005).

8. G. A. Gontcharov, Pisma Astron. Zh. 32, 844 (2006) [Astron. Lett. 32, 759 (2006)].

9. G. A. Gontcharov, Pisma Astron. Zh. 34, 10 (2008) [Astron. Lett. 34, 7 (2008)].

10. L. Girardi, Mon. Not. R. Astron. Soc. 308, 818 (1999).

11. L. Girardi, G. Bertelli, Mon. Not. R. Astron. Soc. 300, 533 (1998).

12. L. Girardi, M.A.T. Groenewegen, A. Weiss, M. Salaris, Mon. Not. R. Astron. Soc. 301, 149 (1998). 
13. L. Girardi, A. Bressan, G. Bertelli, et al., Astron. Astrophys. Suppl. Ser. 141, 371 (2000).

14. L. Girardi, M. Salaris, Mon. Not. R. Astron. Soc. 323, 109 (2001).

15. L. Girardi, M.A.T. Groenewegen, E. Hatziminaoglou, et al., Astron. Astrophys. 436, 895 (2005).

16. E. Høg, C. Fabricius, V.V. Makarov, et al., Astron. Astrophys. 355, L27 (2000).

17. D.J. Marshall, A.C. Robin, C. Reyle, et al., Astron. Astrophys. 453, 635 (2006).

18. S. Nishiyama, T. Nagata, N. Kusakabe, et al., Astrophys. J. 638, 839 (2006).

19. P. P. Parenago, A Course on Stellar Astronomy (GITTL,Moscow, 1954).

20. M. Salaris, L. Girardi, Mon. Not. R. Astron. Soc. 337, 332 (2002).

21. M.F. Skrutskie, R.M. Cutri, R. Stiening, et al., Astron. J. 131, 1163 (2006), http://www.ipac.caltech.edu/2mass/releases/allsky/index.html.

22. M. Steinmetz, T. Zwitter, A. Siebert, et al., Astron. J. 132, 1645 (2006), http://www.rave-survey.aip.de/rave/.

23. C.O. Wright, M.P. Egan, K.E. Kraemer, et al., Astron. J. 125, 359 (2003). 

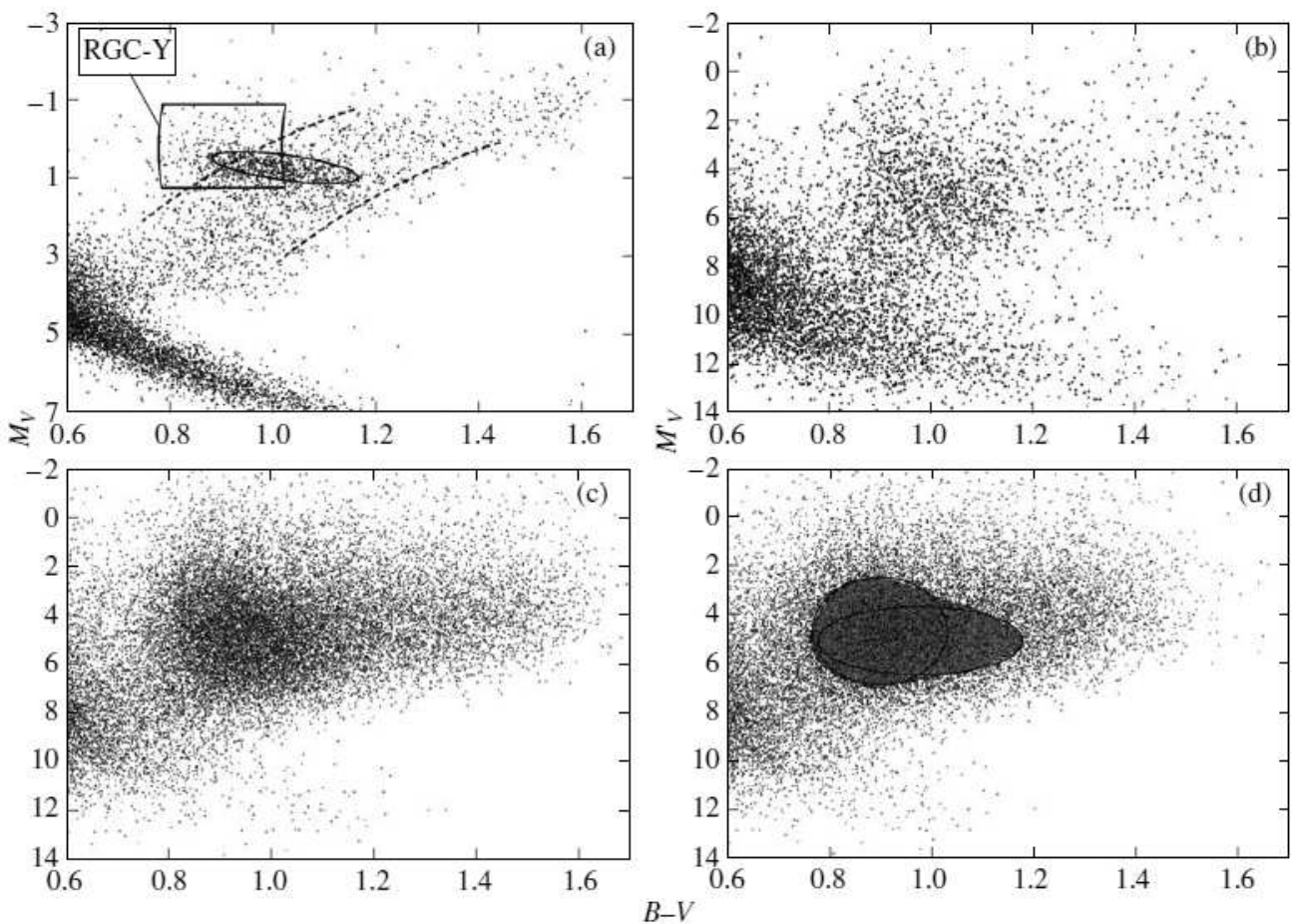

Figure 1: Giants and part of the MS (a) on the $(B-V)-M_{V}$ diagram for Hipparcos stars with the best data, (b) on the $(B-V)$ - reduced proper motion $M_{V}^{\prime}$ diagram for the same Hipparcos stars, (c) on the $(B-V)-M_{V}^{\prime}$ diagram for Tycho-2 stars in a typical sky region before dereddening, and (d) the same after dereddening. The shading marks the region of enhanced distribution density of stars; the two ellipses mark the RGC star selection region. 


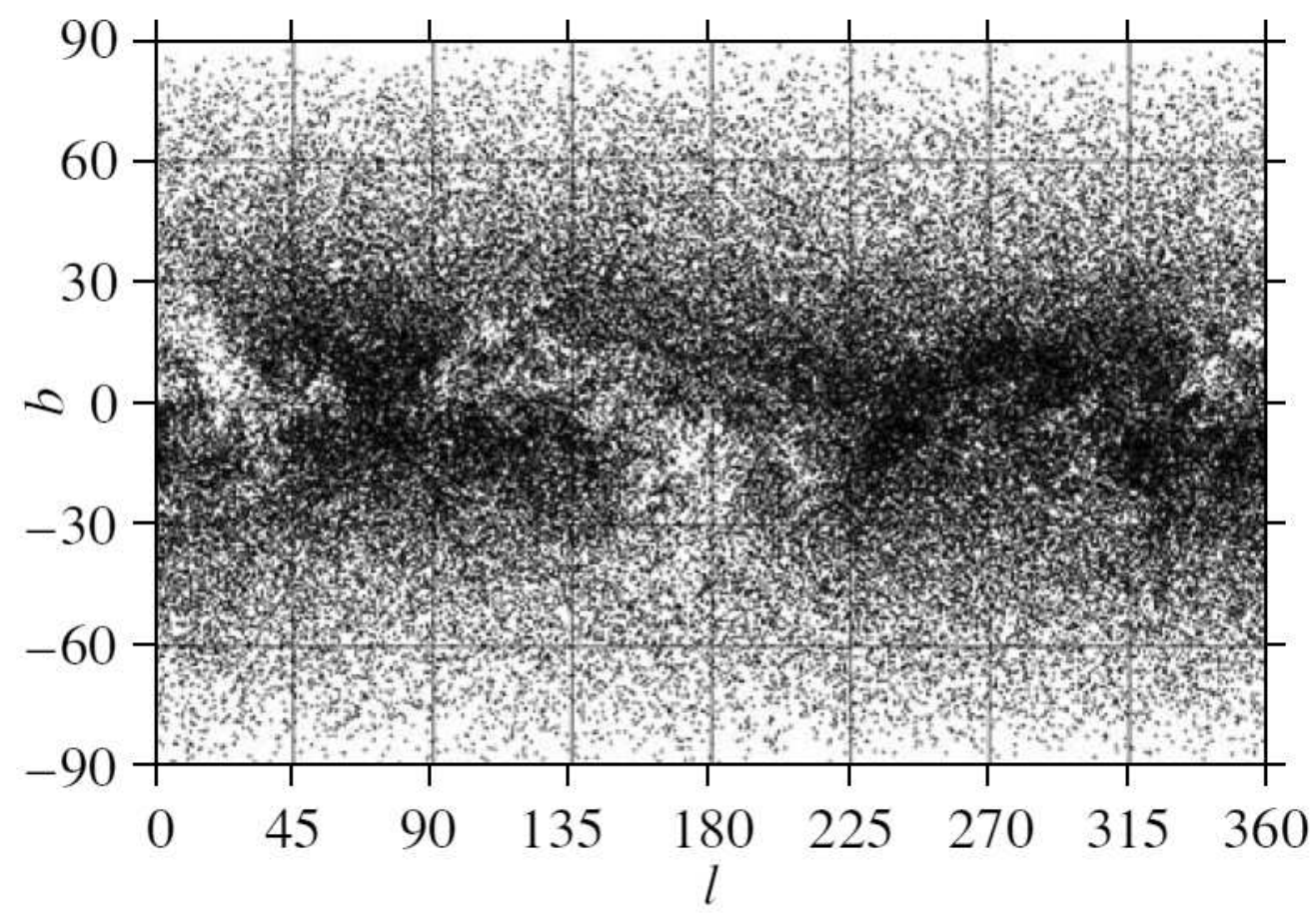

Figure 2: Distribution of 97348 selected presumed RGC stars on the celestial sphere in Galactic coordinates. 

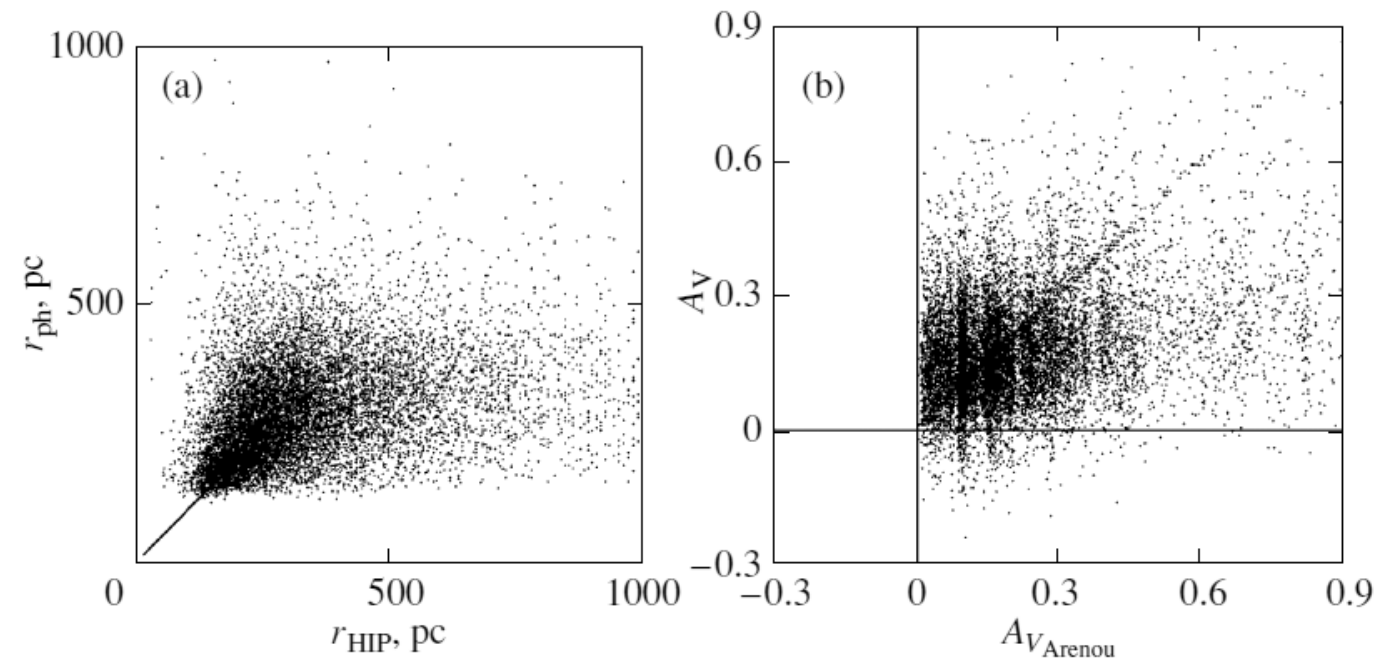

Figure 3: Comparison of the (a) photometric distances with the distances calculated from the Hipparcos parallaxes and (b) the derived extinction with that calculated by Arenou et al. (1992) from the Hipparcos parallaxes for 13 079 selected presumed RGC stars with $\pi>0.001$ arcsec. 


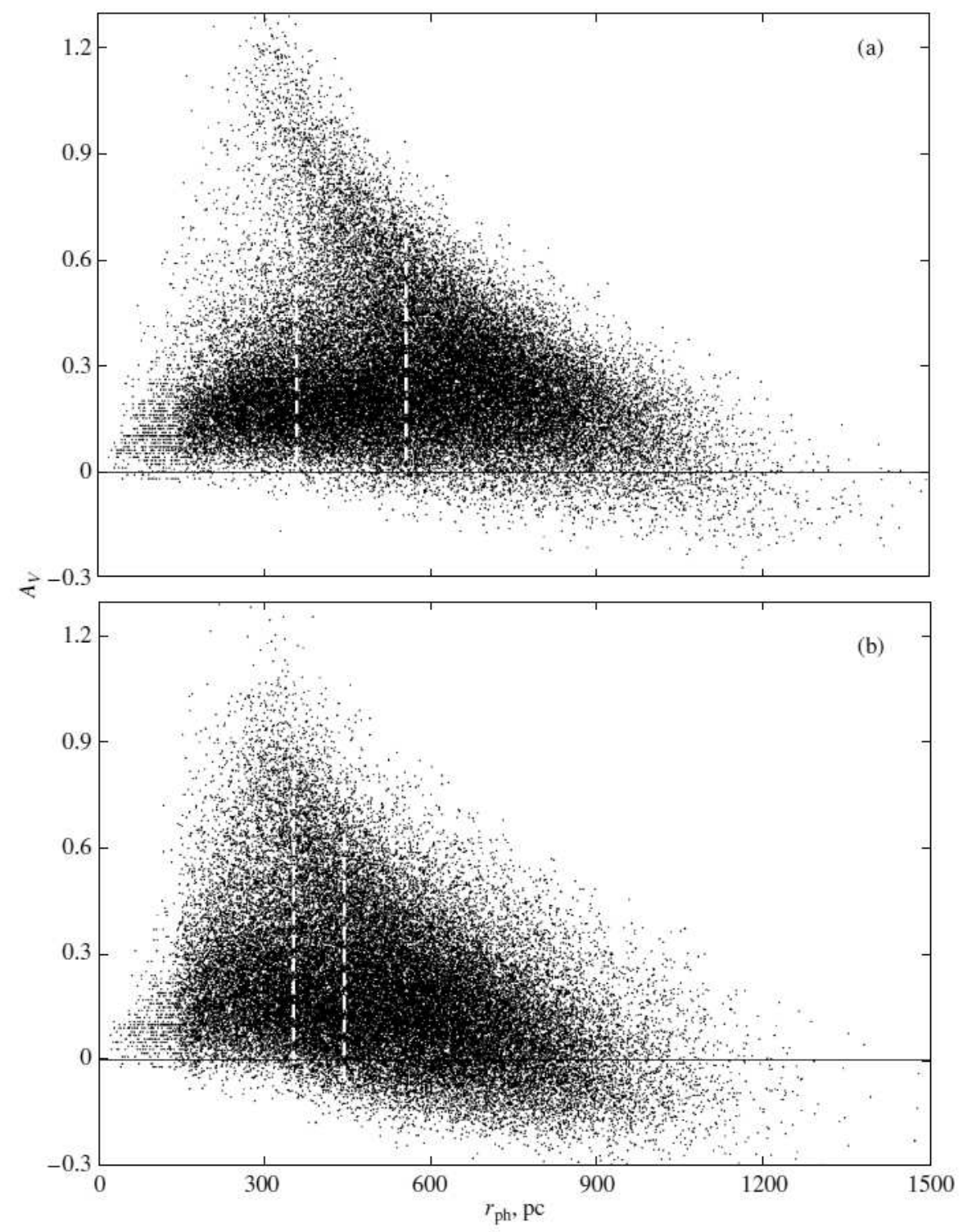

Figure 4: Interstellar extinction $A_{V}$ versus photometric distance for (a) 48 674 selected conditional RGC-Y and (b) conditional RGC-O. 

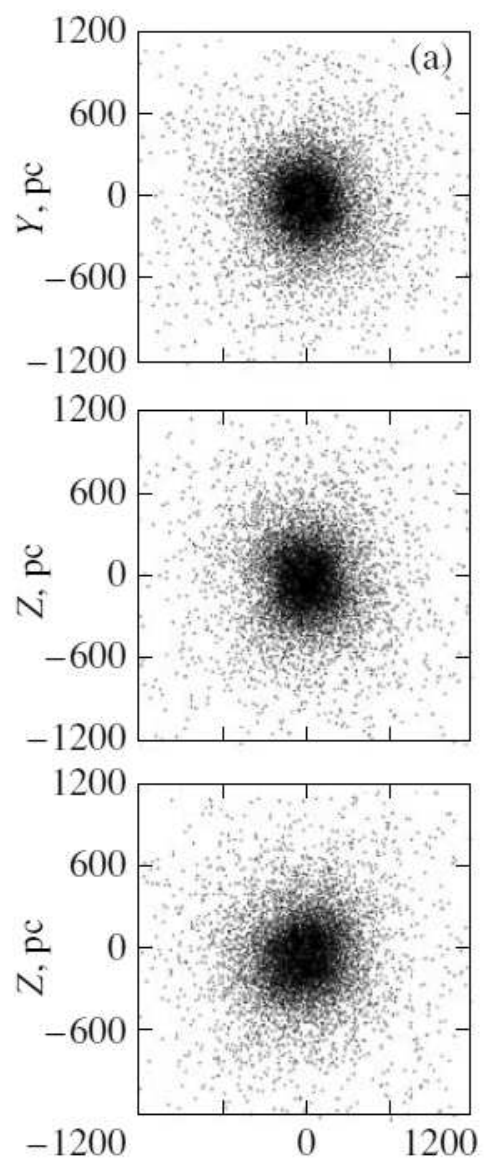
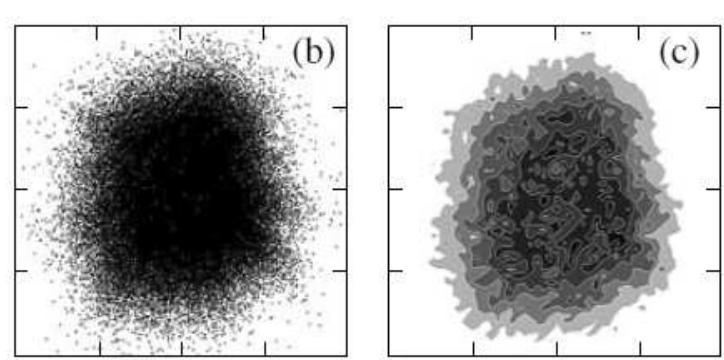

$X, \mathrm{pc}$
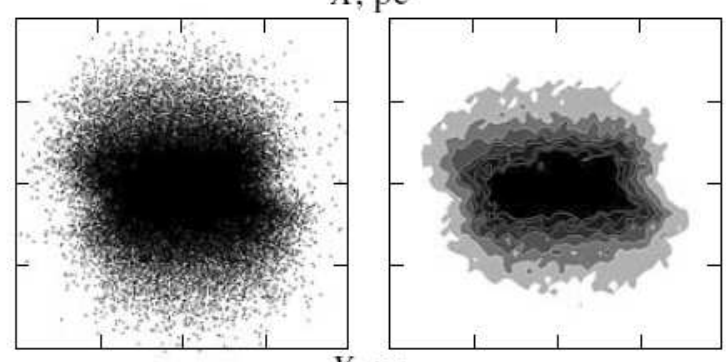

$X, \mathrm{pc}$
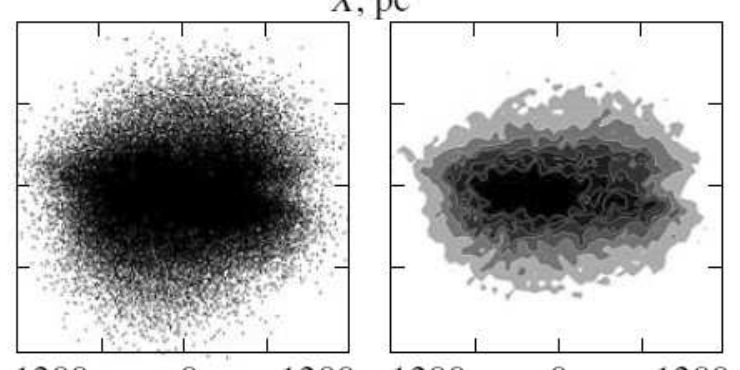

$1200-1200$
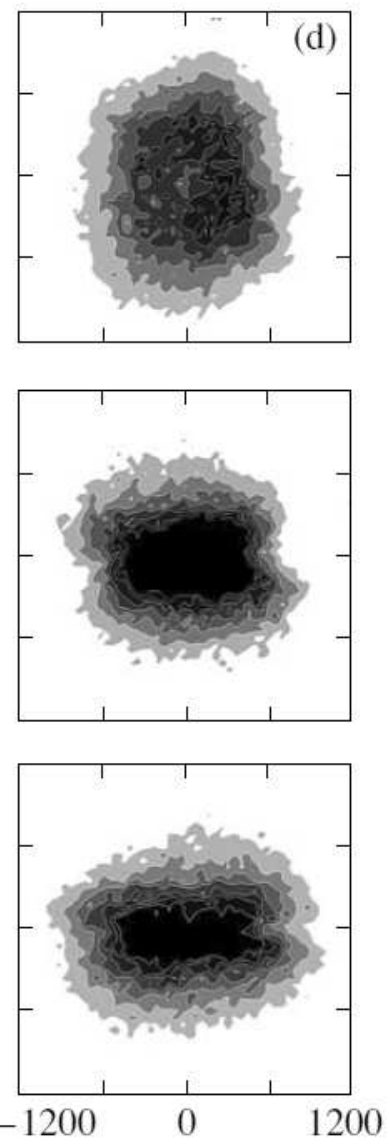

$Y, \mathrm{pc}$

Figure 5: Spatial distributions in projection onto the $X Y, X Z$, and $Y Z$ planes calculated (a) from the Hipparcos parallaxes for 13889 selected presumed RGC stars with positive Hipparcos parallaxes, (b) from the photometric distances for all 97348 selected presumed RGC stars, (c) the same indicated by a smoothed contour map for 48674 conditional RGC-Y, and (d) the same indicated by a smoothed contour map for 48674 conditional RGC-O. 

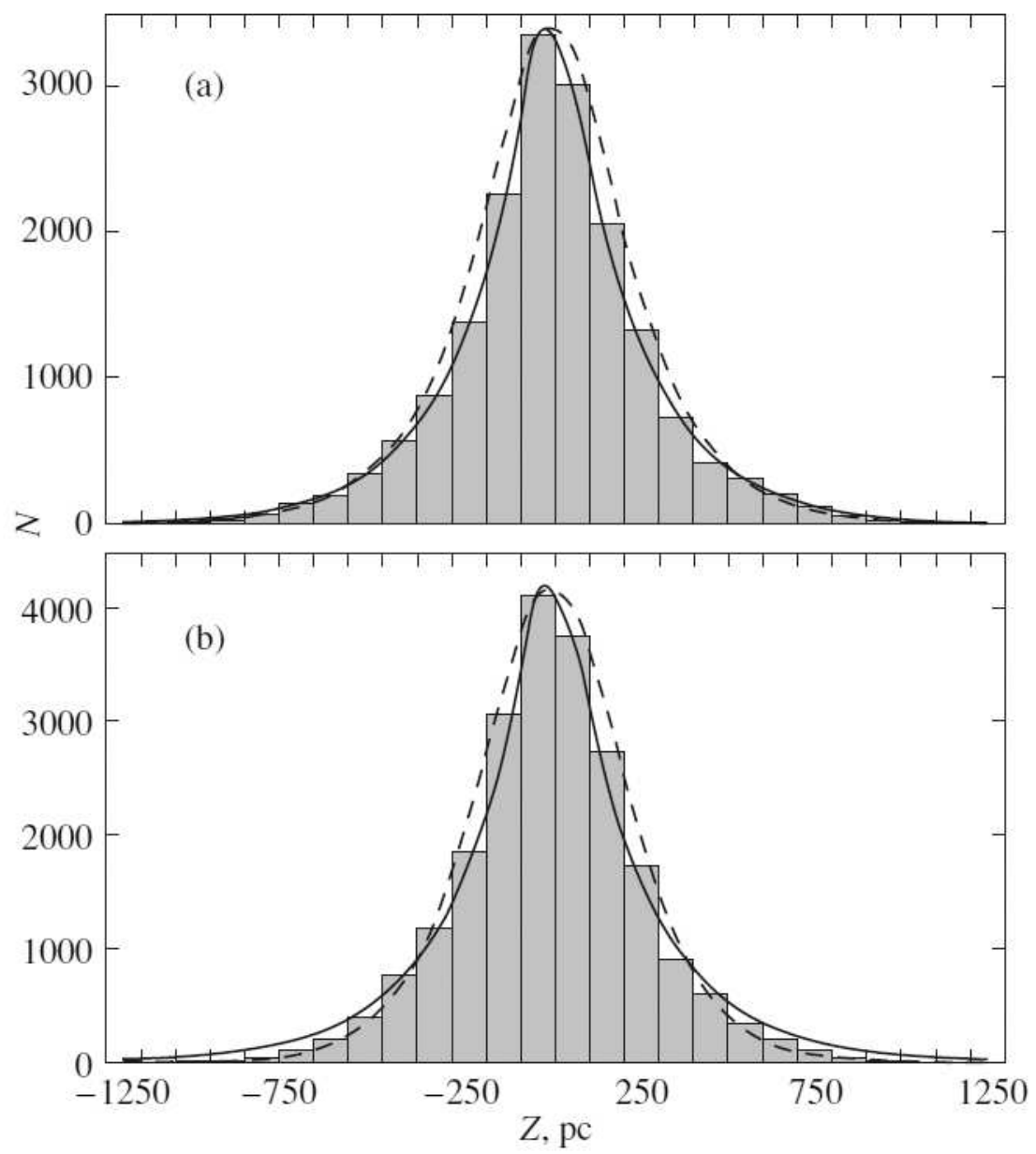

Figure 6: Distributions of (a) 17623 selected conditional RGC-Y and (b) 22304 RGC-O in the vertical cylinder along the $Z$ axis within 400 pc of the Sun; the solid and dotted curves indicate the variations in distribution density according to the barometric law and as the square of the hyperbolic secant, respectively. The asymmetry results from the rise of the Sun above the Galactic plane. 


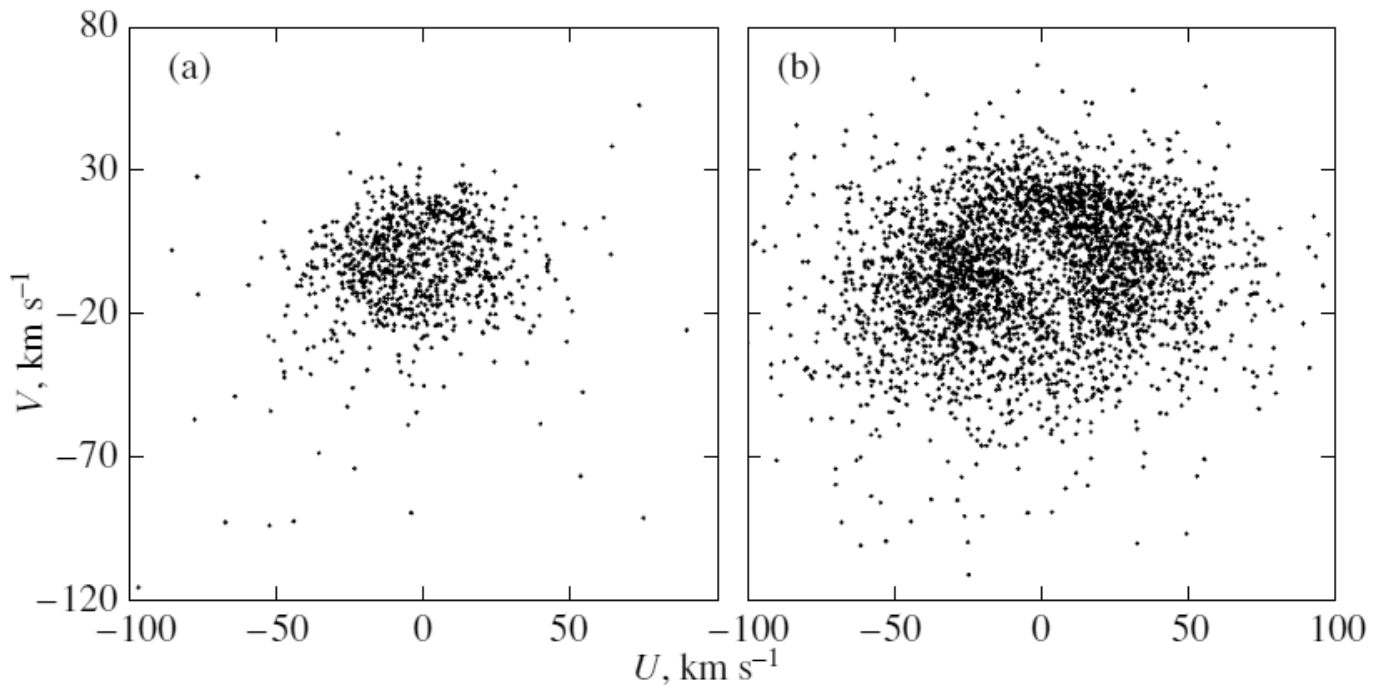

Figure 7: Distribution of the 4163 stars under consideration with radial velocities derived from the velocity components $U$ and $V$ : (a) the presumed 900 youngest and most massive stars and (b) the remaining stars. 